\title{
Clinical values and potential pathways of miR-183-5p in gastric cancer: a study based on integrational bioinformatics analysis
}

\author{
Yanan Wang ${ }^{1 \#}$, Jinku Zhang ${ }^{2 \#}$, Mingkai Liu ${ }^{1}$, Shun Zhang ${ }^{1}$, Weina Wang ${ }^{1}$, Shujie Cheng ${ }^{3}$ \\ ${ }^{1}$ Department of Pathology, Affiliated Hospital of Hebei University, Baoding, China; ${ }^{2}$ Department of Pathology, No. 1 Central Hospital of Baoding, \\ Baoding, China; ${ }^{3}$ Department of Surgery, Affiliated Hospital of Hebei University, Baoding, China \\ Contributions: (I) Conception and design: Y Wang, S Cheng; (II) Administrative support: S Cheng; (III) Provision of study materials or patients: Y \\ Wang, J Zhang, S Cheng; (IV) Collection and assembly of data: J Zhang, M Liu, S Zhang, W Wang, S Cheng; (V) Data analysis and interpretation: J \\ Zhang, M Liu, S Zhang, W Wang, S Cheng; (VI) Manuscript writing: All authors; (VII) Final approval of manuscript: All authors. \\ \#These authors contributed equally to this work. \\ Correspondence to: Shujie Cheng. Department of Surgery, Affiliated Hospital of Hebei University, Baoding 071000, China. Email: csj326647@sina.com.
}

Background: The clinicopathological value and exploration of the potential molecular mechanism of microRNA-183-5p (miR-183-5p) have been investigated in various cancers. This study further explored the transcriptome profile regulated by miR-183-5p.

Methods: Messenger RNA (mRNA) expression data, miRNA expression, and clinical information of stomach adenocarcinoma (STAD) were downloaded from The Cancer Genome Atlas (TCGA) database. Differentially expressed genes (DEGs) that related to mir-189-5p expression and cancer proliferation were acquired using bioinformatics analysis. The biological functions of these genes were analyzed in terms of Gene Ontology (GO) and Kyoto Encyclopedia of Genes and Genomes (KEGG). Hub genes relating to gastric cancer (GC) signal pathway were explored. The results were validated by further experiments.

Results: A total of 308 genes were found to be regulated by miR-183-5p, and they were related to cancer and GC patients' survival outcome. The biological function of these genes was found to act mainly on biological processes and the involved signal pathways included neuroactive ligand-receptor interaction, cell adhesion molecules, and axon guidance. In addition, miR-183-5p was also shown to regulate the mTOR, Wnt, MAPK, and PI3K-Akt signaling pathways through the genes WNT2B, NGFR, and NTRK2.

Conclusions: The miRNA miR-183-5p participates in the tumorigenesis and development of GC via certain signaling pathways, in particular the nerve- and immunity-related genes.

Keywords: Gastric cancer (GC); miR-183-5; neurogene; immunity; population-based evidence

Submitted Aug 26, 2021. Accepted for publication Oct 20, 2021.

doi: 10.21037/jgo-21-599

View this article at: https://dx.doi.org/10.21037/jgo-21-599

\section{Introduction}

Gastric cancer (GC) is the fifth most common cancer with more than 1 million cases in 2018 (1), is the fifth most frequently diagnosed cancer, and the third leading cause of cancer deaths in both men and women worldwide (2). Almost two-thirds of GC cases occur in developing countries (3). MicroRNA (miRNA) is a type of small, non-coding RNA that is involved in post-transcriptional processes and the proliferation, apoptosis, and migration of tumor cells, thus affecting tumorigenesis and the development of tumors (4). The clinicopathological value and potential molecular mechanism of miRNA-183-5p located on chromosome $7 \mathrm{q} 32.2$ has been investigated in various cancers, including bladder cancer (5), lung adenocarcinoma (6), hepatocellular carcinoma $(7,8)$, prostate cancer (9), cervical cancer (10), renal cell cancer (11), ovarian cancer (12), and colorectal cancer (13). High expression levels of miR-183-5p displayed correlation with low rates of overall survival, predictive of worse prognoses of patients with various cancers involving 
pathway including EMT, PTEN and so on, and it has also been reported in GC-involved pathways including Bcl-2/ P53 (14), PI3K/AKT/mTOR (15), Wnt/ $\beta$-catenin (16), and the apoptosis-related signaling pathway. While results have already been obtained from cellular and animal experiments, population statistics and status still need to be explored.

In the present study, we attempted to investigate the clinical significance of the expression level and genetic alteration of microRNA-183-5p (miR-183-5p) in GC based on data from The Cancer Genome Atlas (TCGA; https:// cancergenome.nih.gov/), cBioPortal for Cancer Genomics (http://www.cbioportal.org/), and Gene Expression Omnibus (GEO; https://www.ncbi.nlm.nih.gov/geo/). In addition, we identified potential target genes of miR-183-5p via differentially expressed genes (DEGs) calculated by RNAseq data from TCGA predicting platforms and gene profiling post miR-183-5p overexpression. Further bioinformatic analyses, including the enrichment of functional annotation and biological pathway analyses, were performed to explore the possible roles of miR-183-5p in the tumorigenesis and progression of GC among populations. Our study revealed the transcriptome profile regulated by miR-183-5p in GC. The biological function of the transcriptome profile was also described. It helps to explore the new clinical intervention for GC. We present the following article in accordance with the MDAR reporting checklist (available at https://dx.doi. org/10.21037/jgo-21-599).

\section{Methods}

\section{Patients and datasets}

Messenger RNA (mRNA) expression data, miRNA expression, and clinical information of stomach adenocarcinoma (STAD) were downloaded from TCGA database using the R package TCGA biolinks (17). A total of 450 samples of STAD tumors with complete data were included. The ensemble ID of TCGA samples was annotated with human genome GRCh38/hg38. Due to the data used in this study being collected from public datasets, no participant informed consent or ethical review was required per local policy on the use of public datasets. The study was conducted in accordance with the Declaration of Helsinki (as revised in 2013).

\section{Genes regulated by miR-183-5p}

Using the median classification method, STAD cohorts were divided into 2 groups, high- (206 samples, if the expression of miR-183-5p is higher than the miR-1835 p's median expression value of all samples) and lowexpression (197 samples, if the expression of miR-183-5p is not higher than the miR-183-5p's median expression value of all samples), according to the expression of miR-183-5p. Transcriptome Gene expression comparison between high and low expression groups was conducted using $\mathrm{R}$ package edgeR (3.26.5) (18). The difference screening threshold was set so that false discovery rate (FDR) $<0.05$ and the absolute value of $\log 2$ FoldChange $>1$. The different expression genes between the miR-183-5p high and low expression groups were considered to be regulated by miR-183-5p.

\section{Genes relating to cancer}

After screening out genes regulated by miR-183-5p, we further determined genes relating to cancer from genes regulated by miR-183-5. The STAD cohorts were divided into 2 groups, tumor (415 samples) and benign (35 samples). Gene expression comparison between tumor and benign sample groups was conducted using $\mathrm{R}$ package edgeR (3.26.5) (18). The difference screening threshold was set that FDR was less than 0.05 and the absolute value of Log2FoldChange $>1$.

\section{Survival analysis}

Overall survival (OS) analysis of genes regulated by miR183-5p and relating to cancer was employed by KaplanMeier (KM) plotter analysis (http://kmplot.com/analysis/), and included clinical data and gene expression information from 415 STAD patients (19). Subsequently, information on the number of cases along with median values of mRNA expression levels, hazard ratio (HR) with a $95 \%$ confidence interval (CI), and log-rank $\mathrm{P}$ values were extracted from the KM plotter webpage. Statistical significance was considered when $\mathrm{P}<0.05$.

\section{Biological analysis}

The R package clusterProfiler was used to further explore the biological significance of the genes regulated by miR$183-5$ p, relating to cancer and survival (20). In Gene Ontology (GO) and Kyoto Encyclopedia of Genes and Genomes (KEGG) analysis, FDR $<0.05$ was considered a significant enrichment. 


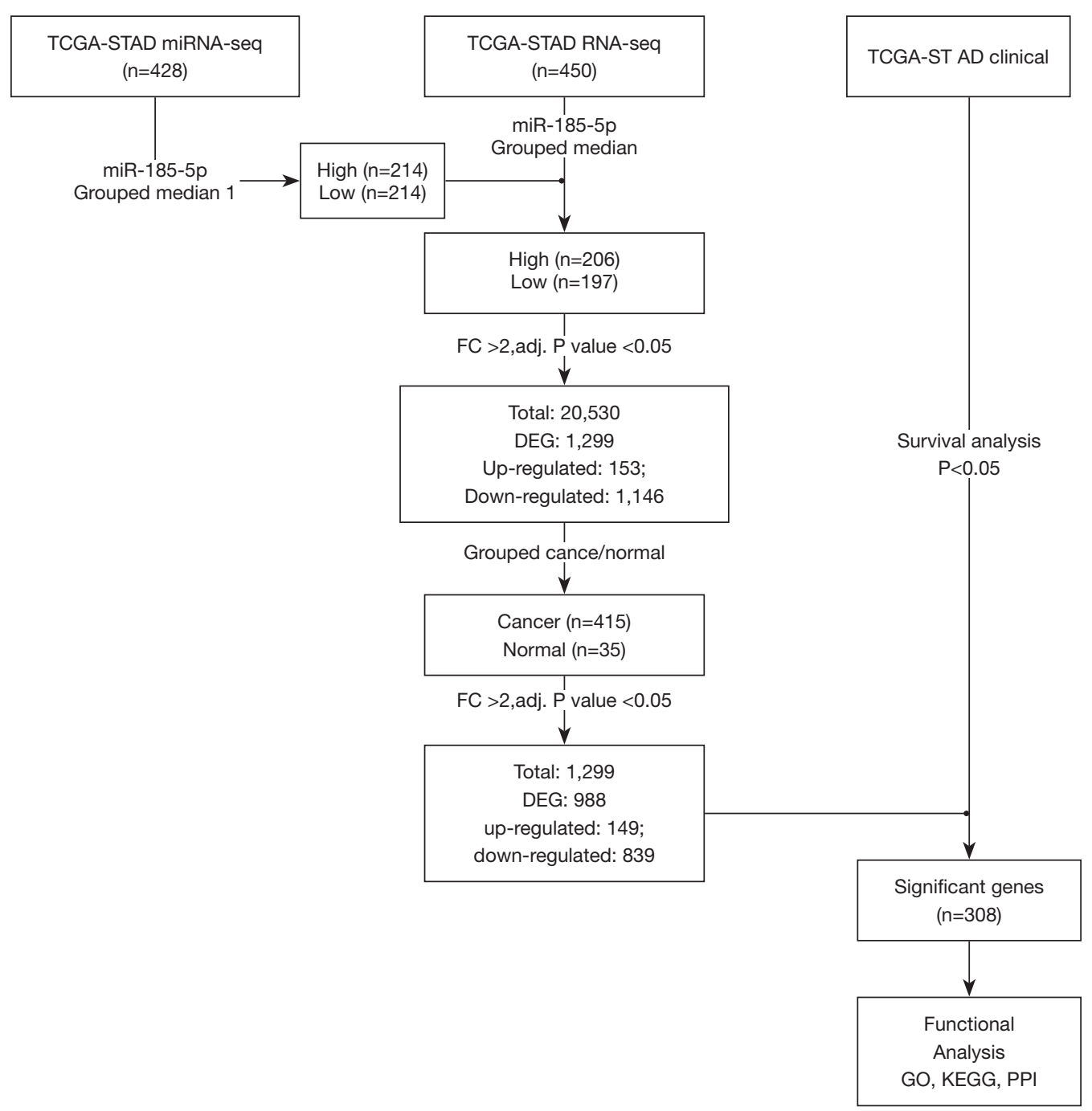

Figure 1 Study design and analysis flowchart. TCGA STAD transcriptome data and clinical data was used. TCGA, The Cancer Genome Atlas; STAD, stomach adenocarcinoma; DEG, differentially expressed gene; FC, fold change; GO, Gene Ontology; PPI, protein-proteininteraction.

\section{Interaction network generation and bub genes analysis}

An interaction network of genes regulated by miR-183-5p relating to cancer and survival was built using the Search Tool for the Retrieval of Interacting Genes (STRING, http://string-db.org/) database (21). A combined score of $\geq 0.4$ was the cut-off value. Cytoscape software (version 3.6.0; https://cytoscape.org/) was used for network visualization (22). According to 12 ranking methods in cytoHubba (23), and APP in Cytoscape, the top 10 genes of each method were selected for the analysis of overlapping genes, and genes with the highest number of overlaps were used as hub genes and potential biomarkers. The whole pipeline is described (Figure 1).

\section{Cell culture and treatments}

We obtained SGC-7901 cells from the American Type Culture Collection (ATCC; Rockville, MD, USA). Short tandem repeat (STR) genotyping was conducted for quality control, with an Axygen DNA extraction kit (Axygen Inc., Corning, NY, USA) used for DNA extraction and ABI3730XL (Thermo Fisher Scientific, Waltham, MA, USA) used for gene analysis. Cells were kept in Dulbecco's 

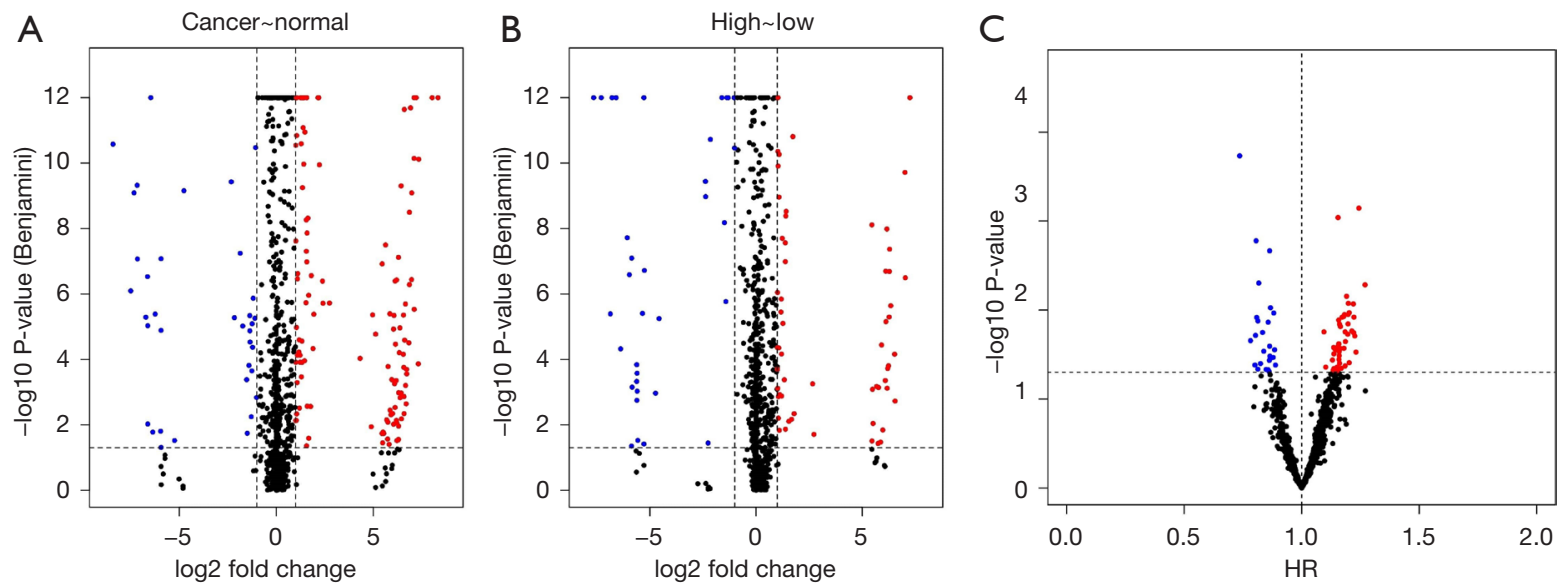

Figure 2 Transcriptional profile of gene expression between sample groups. Volcano plot of DEGs between (A) cancer sample and normal sample, (B) miR-185-5p high expression and low expression groups. (C) Correlation between survival outcomes (HR, hazard ratio) and DEG levels. $x$-axis: fold changes (A,B) or HR (C), y-axis: significance. Up-regulated/down-regulated genes show in red/blue (non-significant genes in black). DEGs, differentially expressed genes.

modified Eagle medium (DMEM)/high glucose medium supplemented with $10 \%$ fetal bovine serum (FBS) and $1 \%$ penicillin $(100 \mathrm{U} / \mathrm{mL}) /$ streptomycin $(0.1 \mathrm{mg} / \mathrm{mL})$ at $37{ }^{\circ} \mathrm{C}$ in a $5 \% \mathrm{CO}_{2}$ humidified culture incubator. The specific miR183-5p mimics and inhibitor (Jima Gene, Shanghai, China) were used to upregulate and inhibit the expression of miR183-5p respectively. The SGC-7901 cells were cultured in a 6 -well plate at $70-80 \%$ confluence for $24 \mathrm{~h}$. The culture medium in each well was then mixed with $1 \mu \mathrm{M}$ of mimics or inhibitor in lipo2000 (Thermo Fisher Scientific, USA). After transfection for $24 \mathrm{~h}$, the expression of target genes NTRK2, NGFR, and WNT2B were confirmed by quantitative polymerase chain reaction (qPCR).

\section{Reverse transcription-qPCR analysis}

Total RNA was extracted from cells after treatment using Trizol (Invitrogen, Carlsbad, CA, USA). Subsequently, RNA was reverse-transcribed using PrimeScript ${ }^{\mathrm{TM}} \mathrm{RT}$ Master Mix (Perfect Real Time) (RR036A, Takara Bio, Kusatsu, Shiga, Japan). We then performed qPCR amplification an ABI step one plus system (Applied Biosystems, Waltham, MA, USA) with PowerUP ${ }^{\text {TM }}$ SYBR $^{\text {TM }}$ Green $^{\circledR}$ Master Mix (A25742, Thermo Fisher, USA). The expression level of the target gene was standardized via glyceraldehyde 3-phosphate dehydrogenase (GAPDH), and $2^{-\Delta \Delta C T}$ was used to calculate the expression results. The gene primers are listed in
Table S1. Each group set 3 duplicate wells.

\section{Statistical analysis}

The compassion between results of laboratory validated experiments were presented as means \pm SD. Student's $t$-test was used to compare means between two groups of Reverse transcription-qPCR. All the statistical analyses were performed using Statistical Product and Service Solutions (SPSS) software (version 19.0). The $\mathrm{P}$ value $<0.05$ is considered to be statistical significance.

\section{Results}

\section{Identification and functional analysis of genes regulated by miR-183-5p, related to cancer and survival}

Participants were divided into high- and low-expression groups according to miR-183-5p expression. The cutoff value was 5.95 . The sample sizes of high- and lowexpression groups were 206 and 197, respectively. Among the 20,530 genes, 1,299 were DEGs. A total of 153 genes were upregulated, while 1,146 genes were downregulated (Figure 2A). Of these genes, 149 in the tumor sample were high expression while 839 were low expression (Figure 2B). Then, we used these 998 genes to calculate their relationship with survival. A total of 308 genes were 

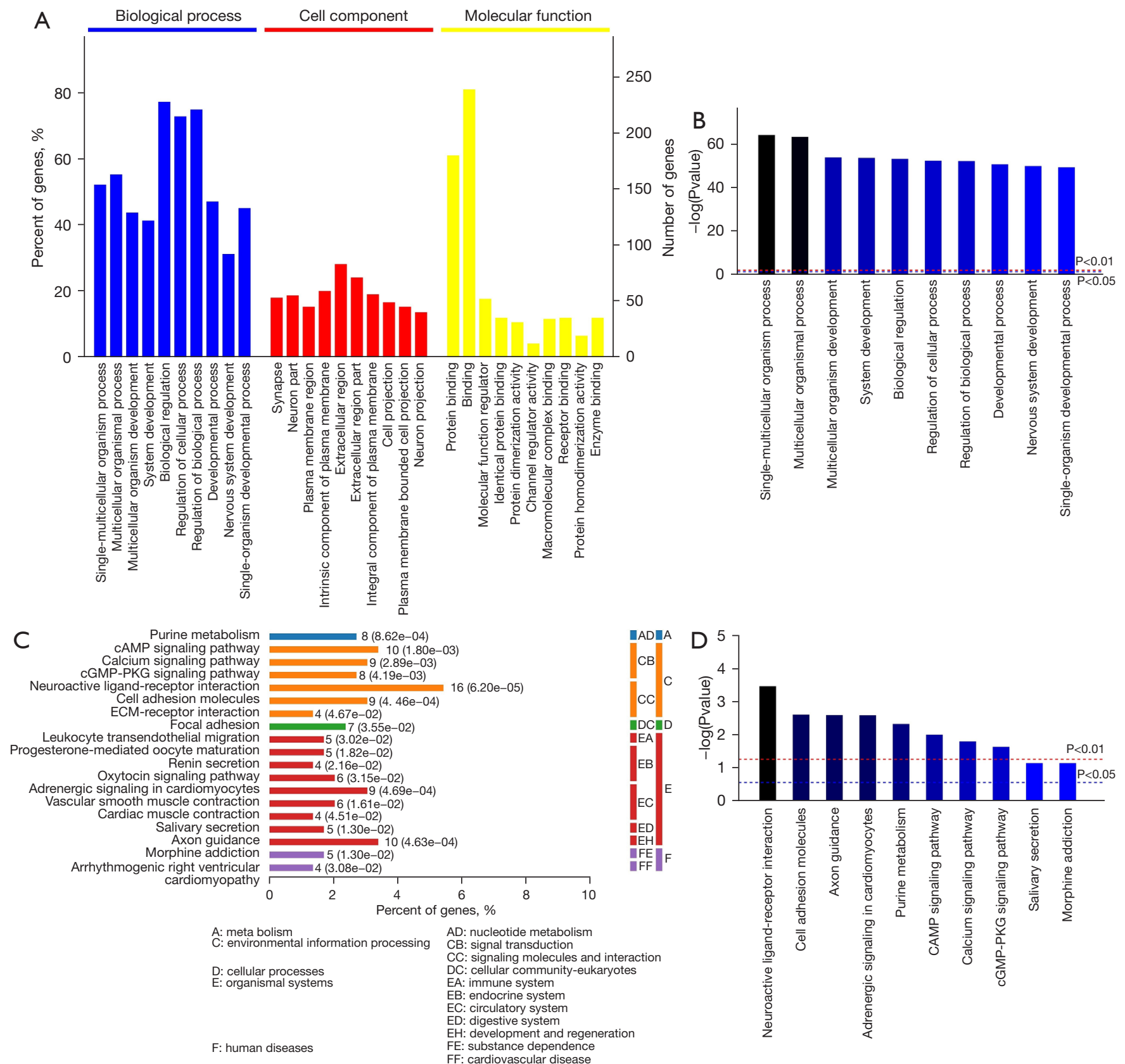

Figure 3 Functional enrichment. (A) DEG enrichment in GO. (B) P value of top ten GO terms. (C) KEGG pathway enrichment. Gene percentage of each pathway shown in bar plot. Pathway types was filled in bar color with detailed annotation list in below. (D) $\mathrm{P}$ value of top 10 KEGG pathways. GO, Gene Ontology; KEGG, Kyoto Encyclopedia of Genes and Genomes.

significantly correlated with different survival outcomes (Figure 2C).

\section{Biological function analysis}

A total of 308 genes were analyzed in terms of GO, including biological process (BP), cell component (CC), and molecular function (MF) and KEGG. The BP mainly included organism process and development; CC included extracellular region, plasma membrane region, cell projection, and so on; MF included protein binding, 
molecular function regulators, and so on (Figure $3 A$ ). The top 10 significant GO terms were mainly in $\mathrm{BP}$ and involved the organism processes and the development and regulation of cellular processes (Figure 3B). Neuroactive ligand-receptor interaction, cell adhesion molecules, axon guidance, and so on were the first 3 enriched pathways regulated by miR-183-5p (Figure 3C,3D).

\section{Interaction network generation analysis}

A total of 308 targeted genes were analyzed using STRING. We found that the mTOR, Wnt, MAPK, and PI3K-Akt signaling pathways that relate to GC were influenced by genes regulated by miR-183-5p. Involved genes are listed in available online: https://cdn.amegroups.cn/static/ public/jgo-21-599-1.xlsx. The PI3K-Akt signaling pathway involved 7 genes and the MAPK signaling pathway involved 6 genes. The mTOR signaling pathway was connected by WNT2B. Among these genes, NTRK2, NGFR, and $W N T 2 B$ were connectors (Figure 4).

\section{Validation experiments}

After transfection with miR-183-5p mimics and inhibitor, mir-183-5p was up-regulated by 155.3 -fold $(\mathrm{P}<0.05)$ and down-regulated by 0.00641 -fold $(\mathrm{P}<0.001)$ by qPCR, respectively. After miR-183-5p mimics was used, the expressions of NTRK2, NGFR, and $W N T 2 B$ were inhibited. The expressions of NTRK2, NGFR, and $W N T 2 B$ were promoted after the miR-183-5p inhibitor was used. The expressions of NTRK2, NGFR, and $W N T 2 B$ were lower in tumor tissue than in benign tissue (Figure 5).

\section{Discussion}

Mediation by miRNA regulates cell growth and apoptosis and is involved in cancer (24) through negatively regulated gene expression (25), thereby resulting in changes in cell development (26), proliferation (27), and apoptosis $(28,29)$. It has been reported that miR-183-5p is abnormally expressed in some types of cancer and exerts significant effects on the proliferation, apoptosis, drug resistance, and metastasis of cancer cells (30). It modulates the chemosensitivity of human non-small cell lung cancer to cisplatin via targeting ABCC1 (31). Currently, in vitro experiments have shown that miR-185-5p promotes apoptosis and regulates autophagy in GC $(15,30)$, activates the transforming growth factor- $\beta$ (TGF- $\beta$ ) pathway in osteosarcoma, and activates the $\mathrm{Wnt} / \beta$-catenin signaling pathway in melanoma (16).

In this study, we further explored the biological role of miR-183-5p using a population dataset. Our results showed that miR-185-5p was significantly up-regulated in the tumor group compared to the normal group. After being screened from the population, 308 genes were found to be regulated by miR-183-5p and were related to cancer and GC patients' survival outcomes. The GO biological function acted mainly on biological processes relating to single organism development, single-multicellular organism process, and development. In addition, miR-183-5p was shown to influence the binding of proteins and other molecules. Stem cells are highly correlated with cell and tissue development. Sponging miR-183-5p promotes osteosarcoma growth and metastasis by regulation of stem cells (30). Our results showed that miR-183-5p potentially regulates stem cells, a hypothesis which requires further experiments to elucidate. In terms of KEGG, the enrichment pathway mainly focused on neuroactive ligand-receptor interaction, immune system, and development and regeneration. The relationship between the nervous system, immunity, and cancer has been discussed previously. This relationship can be summarized as: severe physical or mental stress $\rightarrow$ sympathetic nerve activation $\rightarrow$ granulocytosis $\rightarrow$ tissue damage. Tissue damage includes collagen diseases, inflammatory bowel diseases, and cancer. In addition, granulocytes in the gastric mucosa might be important in the pathogenesis of gastric ulcers (32). Faulkner et al. reported that nerves are emerging regulators of cancer initiation, progression, and metastasis (33). The GO and KEGG results provided evidence that miR$183-5 \mathrm{p}$ can regulate nervous and immunity-related pathways to inhibitor development of GC.

Previous in vitro studies have reported PI3K-Akt/mTOR and MAPK signaling pathways in GC $(15,34)$, and that miR-183-5p regulates Wnt signaling in bladder cancer (35). Our results indicated that the PI3K-Akt/mTOR and Wnt signaling pathways in GC were regulated by miR-183-5p in the population dataset. In addition, we also reported the key genes that involve these 4 signal pathways. Among these genes, NRTK2 and NGFR were connectors between the PI3K-Akt and MAPK signaling pathways. These 2 genes are neurogenes and potential paths through which miR183-5p plays its regulatory role. The mitogen-activated protein kinase MAPK10 is the connector between the WNT and MAPK signaling pathways, and $W N T 2 B$ is 


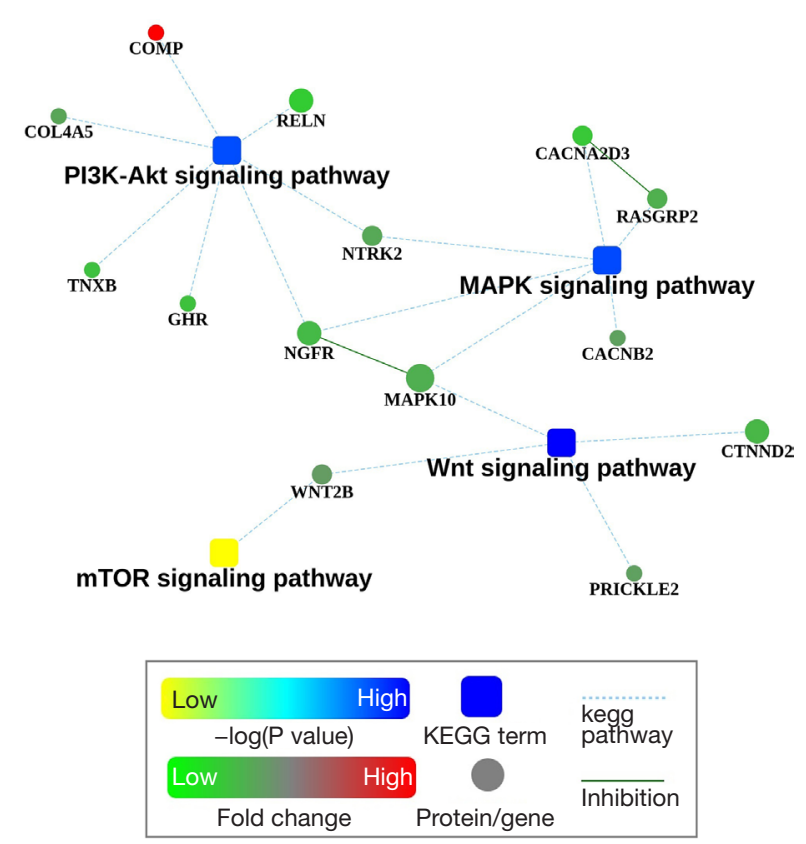

Figure 4 Gene-pathway interaction network. KEGG pathway shown in rectangles, filled by enrichment $\mathrm{P}$ value (significant level increase from yellow to blue). Protein-coding genes shown in circles, filled by FC (change level increase from green to red). Protein-protein interaction marked by solid lines. Genes in pathway linked by dashed lines. KEGG, Kyoto Encyclopedia of Genes and Genomes; FC, fold change.

the connector between the WNT and mTOR signaling pathways. When regulated by miR-185-3p in vitro, WNT2B has shown radioresistance in nasopharyngeal carcinoma (36). These 3 genes were significantly down-regulated in the tumor group compared to the normal group. Compared to the low expression group of miR-185-5p, NTRK2, $N G F R$, and $W N T 2 B$ in the high expression group were all significantly downregulated. The results were validated by further experiments.

In this study, we further explored the biofunction of miR-185-3p based on the GC population dataset. Based on consistent previous results in vitro, we found pathways reported in other cancers in GC. In addition, this study found that miR-185-3p influences the organism process and development and regulation of cellular processes, and plays its regulatory role through neurogenes in GC.

\section{Conclusions}

In this study, we analyzed the transcriptome profile

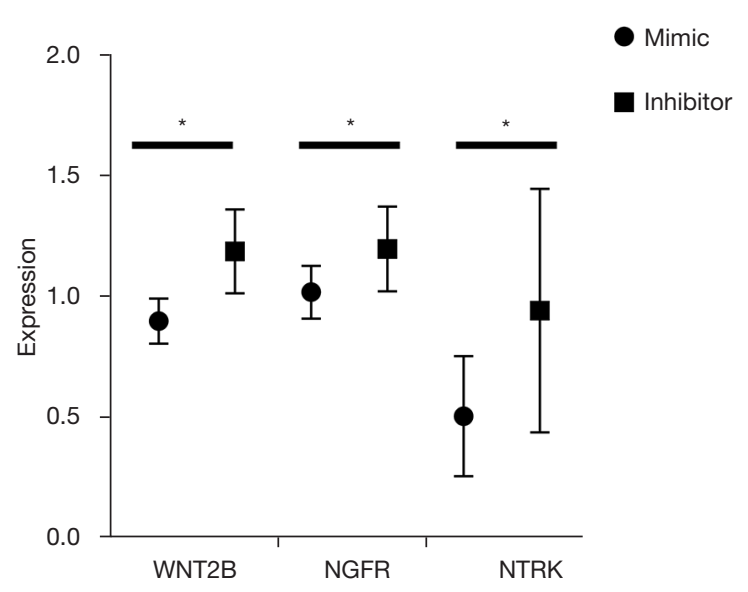

Figure 5 miR-185-5p validation. Rectangles: miRNA inhibitor; circles: miRNA mimic; asterisk: statistical significance between mimic and inhibitor induced gene (x-axis) expressions changes (y-axis). miRNA, microRNA.

regulated by miR-183-5p in GC. Then we furtherly described the biological function of this transcriptome profile. Use the network analyzed method, this study reveals the relationship among multiple cancer-related signaling pathways of mTOR, Wnt, MAPK, and PI3K-Akt. Three related potential biomarkers were found and validated by experiment. Our work can help to support the generation of new clinical intervention for GC.

\section{Acknowledgments}

Funding: This work was supported by the Department of Science and Technology of Hebei Province (162777241).

\section{Footnote}

Reporting Checklist: The authors have completed the MDAR reporting checklist. Available at https://dx.doi. org/10.21037/jgo-21-599

Conflicts of Interest: All authors have completed the ICMJE uniform disclosure form (available at https://dx.doi. org/10.21037/jgo-21-599). The authors have no conflicts of interest to declare.

Ethical Statement: The authors are accountable for all aspects of the work in ensuring that questions related to the accuracy or integrity of any part of the work are appropriately investigated and resolved. The study was 
conducted in accordance with the Declaration of Helsinki (as revised in 2013). Institutional ethical approval and informed consent were waived.

Open Access Statement: This is an Open Access article distributed in accordance with the Creative Commons Attribution-NonCommercial-NoDerivs 4.0 International License (CC BY-NC-ND 4.0), which permits the noncommercial replication and distribution of the article with the strict proviso that no changes or edits are made and the original work is properly cited (including links to both the formal publication through the relevant DOI and the license). See: https://creativecommons.org/licenses/by-nc-nd/4.0/.

\section{References}

1. Bray F, Ferlay J, Soerjomataram I, et al. Global cancer statistics 2018: GLOBOCAN estimates of incidence and mortality worldwide for 36 cancers in 185 countries. CA Cancer J Clin 2018;68:394-424.

2. Venerito M, Ford AC, Rokkas T, et al. Review: Prevention and management of gastric cancer. Helicobacter 2020;25 Suppl 1:e12740.

3. Thrift AP, El-Serag HB. Burden of Gastric Cancer. Clin Gastroenterol Hepatol 2020;18:534-42.

4. He B, Zhao Z, Cai Q, et al. miRNA-based biomarkers, therapies, and resistance in Cancer. Int J Biol Sci 2020;16:2628-47.

5. Gao JM, Huang LZ, Huang ZG, et al. Clinical value and potential pathways of miR-183-5p in bladder cancer: A study based on miRNA-seq data and bioinformatics analysis. Oncol Lett 2018;15:5056-70.

6. He RQ, Gao L, Ma J, et al. Oncogenic role of miR-183-5p in lung adenocarcinoma: A comprehensive study of qPCR, in vitro experiments and bioinformatic analysis. Oncol Rep 2018;40:83-100.

7. Duan X, Li W, Hu P, et al. MicroRNA-183-5p contributes to malignant progression through targeting PDCD4 in human hepatocellular carcinoma. Biosci Rep 2020;40:BSR20201761.

8. Yan R, Li K, Yuan D, et al. miR-183-5p promotes proliferation and migration in hepatocellular carcinoma by targeting IRS1 and its association with patient survival. Int J Biol Markers 2020;35:83-9.

9. Hua X, Liu Z, Zhou M, et al. LSAMP-AS1 binds to microRNA-183-5p to suppress the progression of prostate cancer by up-regulating the tumor suppressor DCN. EBioMedicine 2019;50:178-90.
10. Tang Q, Liu L, Zhang H, et al. Regulations of miR-183$5 \mathrm{p}$ and Snail-Mediated Shikonin-Reduced EpithelialMesenchymal Transition in Cervical Cancer Cells. Drug Des Devel Ther 2020;14:577-89.

11. Li H, Pan X, Gui Y, et al. Upregulation of miR-183-5p predicts worse survival in patients with renal cell cancer after surgery. Cancer Biomark 2019;24:153-8.

12. Guo R, Qin Y. LEMD1-AS1 Suppresses Ovarian Cancer Progression Through Regulating miR-183-5p/TP53 Axis. Onco Targets Ther 2020;13:7387-98.

13. Zheng S, Zhong YF, Tan DM, et al. miR-183-5p enhances the radioresistance of colorectal cancer by directly targeting ATG5.J Biosci 2019;44:92.

14. Lin J, Shen J, Yue H, et al. miRNA-183-5p.1 promotes the migration and invasion of gastric cancer AGS cells by targeting TPM1. Oncol Rep 2019;42:2371-81.

15. Li H, He C, Wang X, et al. MicroRNA-183 affects the development of gastric cancer by regulating autophagy via MALAT1-miR-183-SIRT1 axis and PI3K/AKT/mTOR signals. Artif Cells Nanomed Biotechnol 2019;47:3163-71.

16. Chen X, Gao J, Yu Y, et al. RETRACTED: Long noncoding RNA UCA1 targets miR-185-5p and regulates cell mobility by affecting epithelial-mesenchymal transition in melanoma via $\mathrm{Wnt} / \beta$-catenin signaling pathway. Gene 2018;676:298-305.

17. Colaprico A, Silva TC, Olsen C, et al. TCGAbiolinks: an $\mathrm{R} /$ Bioconductor package for integrative analysis of TCGA data. Nucleic Acids Res 2016;44:e71.

18. Robinson MD, McCarthy DJ, Smyth GK. edgeR: a Bioconductor package for differential expression analysis of digital gene expression data. Bioinformatics 2010;26:139-40.

19. Györffy B, Surowiak P, Budczies J, et al. Online survival analysis software to assess the prognostic value of biomarkers using transcriptomic data in non-small-cell lung cancer. PLoS One 2013;8:e82241.

20. Yu G, Wang LG, Han Y, et al. clusterProfiler: an R package for comparing biological themes among gene clusters. OMICS 2012;16:284-7.

21. Szklarczyk D, Franceschini A, Wyder S, et al. STRING v10: protein-protein interaction networks, integrated over the tree of life. Nucleic Acids Res 2015;43:D447-52.

22. Shannon P, Markiel A, Ozier O, et al. Cytoscape: a software environment for integrated models of biomolecular interaction networks. Genome Res 2003; 13:2498-504.

23. Chin $\mathrm{CH}$, Chen $\mathrm{SH}, \mathrm{Wu} \mathrm{HH}$, et al. cytoHubba: identifying hub objects and sub-networks from complex 
interactome. BMC Syst Biol 2014;8 Suppl 4:S11.

24. Hutvágner G, Zamore PD. A microRNA in a multiple-turnover RNAi enzyme complex. Science 2002;297:2056-60.

25. Matsubara H, Takeuchi T, Nishikawa E, et al. Apoptosis induction by antisense oligonucleotides against miR-17-5p and miR-20a in lung cancers overexpressing miR-17-92. Oncogene 2007;26:6099-105.

26. Iorio MV, Ferracin M, Liu CG, et al. MicroRNA gene expression deregulation in human breast cancer. Cancer Res 2005;65:7065-70.

27. Zhao L, Zhang Y, Liu J, et al. miR-185 Inhibits the Proliferation and Invasion of Non-Small Cell Lung Cancer by Targeting KLF7. Oncol Res 2019;27:1015-23.

28. Cheng AM, Byrom MW, Shelton J, et al. Antisense inhibition of human miRNAs and indications for an involvement of miRNA in cell growth and apoptosis. Nucleic Acids Res 2005;33:1290-7.

29. Ambros V. The functions of animal microRNAs. Nature 2004;431:350-5

30. Fan L, Tan B, Li Y, et al. Upregulation of miR-185 promotes apoptosis of the human gastric cancer cell line MGC803. Mol Med Rep 2018;17:3115-22.

31. Pei K, Zhu JJ, Wang CE, et al. MicroRNA-185-5p

Cite this article as: Wang Y, Zhang J, Liu M, Zhang S, Wang W, Cheng S. Clinical values and potential pathways of miR-183-5p in gastric cancer: a study based on integrational bioinformatics analysis. J Gastrointest Oncol 2021;12(5):21232131. doi: 10.21037/jgo-21-599 modulates chemosensitivity of human non-small cell lung cancer to cisplatin via targeting ABCC1. Eur Rev Med Pharmacol Sci 2016;20:4697-704.

32. Abo T, Kawamura T. Immunomodulation by the autonomic nervous system: therapeutic approach for cancer, collagen diseases, and inflammatory bowel diseases. Ther Apher 2002;6:348-57.

33. Faulkner S, Jobling P, March B, et al. Tumor Neurobiology and the War of Nerves in Cancer. Cancer Discov 2019;9:702-10.

34. Shi Y, Sun H. Down-regulation of lncRNA LINC00152 Suppresses Gastric Cancer Cell Migration and Invasion Through Inhibition of the ERK/MAPK Signaling Pathway. Onco Targets Ther 2020;13:2115-24.

35. Chen D, Li SG, Chen JY, et al. MiR-183 maintains canonical Wnt signaling activity and regulates growth and apoptosis in bladder cancer via targeting AXIN2. Eur Rev Med Pharmacol Sci 2018;22:4828-36.

36. Li G, Wang Y, Liu Y, et al. miR-185-3p regulates nasopharyngeal carcinoma radioresistance by targeting WNT2B in vitro. Cancer Sci 2014;105:1560-8.

(English Language Editor: J. Jones) 
Supplementary

Table S1 Primer sequence

\begin{tabular}{lll}
\hline ID & Primer & Sequence (5' to 3') \\
\hline WNT2B & WNT2B-F & TGGATGCCAAGGAGAAGAGGCT \\
WNT2B & WNT2B-R & GTACAGGAACCACTCACGCCAT \\
NGFR & NGFR-F & CCTCATCCCTGTCTATTGCTCC \\
NGFR & NGFR-R & GTTGGCTCCTTGCTTGTTCTGC \\
NTRK2 & NTRK2-F & ACAGTCAGCTCAAGCCAGACAC \\
NTRK2 & NTRK2-R & GTCCTGCTCAGGACAGAGGTTA \\
\hline
\end{tabular}

